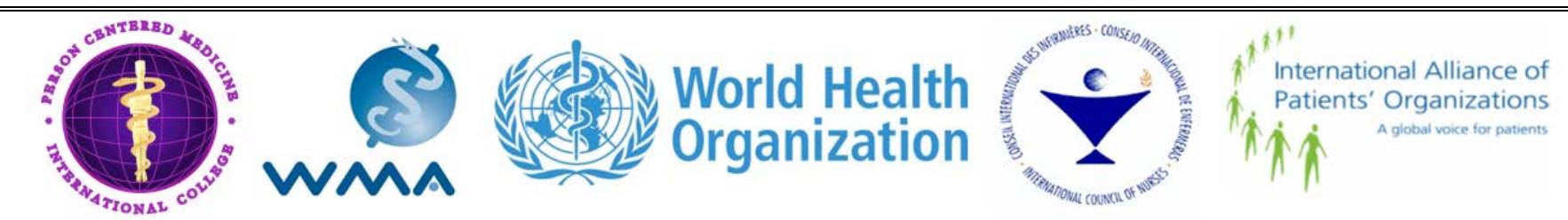

\title{
8th GENEVA CONFERENCE ON PERSON-CENTERED MEDICINE
}

\section{Person-Centered Primary Health Care}

Core Conference on April 27 - 29, 2015

Pre-Conference Work Meetings on April 26, 2015

\section{Geneva University Hospital and World Health Organization CONFERENCE PROGRAM OUTLINE}

\begin{abstract}
Organizing Committee: James Appleyard (President, International College of Person Centered Medicine), Juan E. Mezzich (Secretary General, International College of Person Centered Medicine), Ted Epperly (Program Director for the $8^{\text {th }}$ Geneva Conference on Person-Centered Medicine), Michel Botbol (World Psychiatric Association Psychoanalysis in Psychiatry Section), Tesfamicael Ghebrehiwet (Former Officer, International Council of Nurses), Jo Groves (Former Executive Director, International Alliance of Patients' Organizations), Ihsan Salloum (World Psychiatric Association Classification Section), Sandra Van Dulmen (Former Officer, European Association for Communication in Healthcare), Nuria Toro Polanco (WHO), E. Kelley (WHO), and Hernan Montenegro (WHO).
\end{abstract}

Conference Participants will be clinicians and scholars in medicine and other health fields as well as other interested individuals. ICPCM Continuing Professional Development (CME) Certificates will be e-mailed upon request to all registered participants. The registration fee is 400 Euros for persons residing in World Bank Group A (High Income Countries) and 250 Euros for persons in other countries. Full time students and official representatives of patient and family organizations will pay discounted half rates.

Presentation Formats will include Lectures, Symposia, Interactive Workshops, Brief Oral Presentations and Poster Presentations. Abstracts are due March 31, 2015. Each submission should include a title, authors with location and e-mail address, and an abstract text of up to 200 words and two references.

Conference Secretariat: For further information as well as Registration and Abstract Forms, please visit www.personcenteredmedicine.org and write to the ICPCM Secretariat at Int'l Center for Mental Health, Mount Sinai School of Medicine, Fifth Ave \& 100 St, Box 1093, New York NY 10029, USA. E: ICPCMsecretariat@aol.com.

PRE-CONFERENCE WORKMEETINGS ON PERSON-CENTERED MEDICINE: SUNDAY APRIL 26, 2015

Venue: Auxilliary Halls one floor under the Main, 4 rue Gabrielle-Perret-Gentil CH-1211 Geneva 4, Switzerland.

1:00 - 5:00 PM Pre Conference Institutional Work Meetings:

a. Person-Centered Diagnosis

b. Person-Centered Clinical Care Guiding Principles

c. 2015 Geneva Declaration Draft Presentation

d. Books Workgroup

e. Global Research Network

f. Other Workgroups

5:00 - 6:00 PM IJPCM Editorial Board Meeting

6:00 - 7:00 PM ICPCM Board Meeting 
8:00 - 8:30 AM Registration and check-in

8:30-8:45 AM Conference Opening

Welcoming words from officers of the ICPCM, Geneva University, and World Health Organization

8:45 - 9:15 AM

Keynote Presentation: "Person-Centered Primary Health Care (PCPHC): Now More Than Ever"

(Organizational Note for all Sessions: Each of the regulat sessions would have 3-5 scholatly presentations (all typically involving systematic literature reviews or data-based studies) made by 1-2 core invitees, 1-2 WHO or WMA reps, 0-1 specially supported invitees, and 1-2 non-supported contributors selected from those submitting abstracts on their own.)(Each presentation 8 minutes followed by discussion 7 minutes, and general discussion and conclusions at the end)

9:15 - 10:15 AM Plenary Symposium 1: WHO and International Experience and Perspectives on Person- and People-Centered Primary Health Care

- The WHO Perspective on Primary Care

- Interpersonal universal care: all together in dignity

- The Triple Aim and How Primary Care Achieves This

- How Does Person Centeredness Work?

10:15 - 10:45 AM $\quad$ Coffee Break and Poster Session

10:45 - 12:15 PM Plenary Symposium 2: PCPHC Throughout the Life Cycle

- WHO Perspectives on Ageing and Health

- PCPHC and Obstetrical Care

- PCPHC in Pediatric Care

- Personalized Care Planning for Adults with Chronic Health Conditions

- More Good Days and Shared Decision Making at the End of Life

12:15-1:15PM $\quad$ Lunch (open)

1:15 - 2:45 PM Parallel Sessions 1:

A: Health Care Integration

- What is a Patient Centered Medical Home PCMH?

- What is the PCMH Neighborhood?

- What is an Accountable Care Organization?

- Primary care and integration of health and social services

- Introducing individual and family continuous records for person-centered care

B. Clinical Communication

- Basic Communication

- How People as Patients Want to be Spoken to?

- Shared Decision Making and Primary Care

- Communication in Cancer Care

- Communication across cultures

2:45 - 3:00 PM $\quad$ Coffee Break

3:00 - 4:30 PM Parallel Sessions 2:

A. The Linking of Medical Specialties and Health Care Disciplines in PCPHC

- How Should Specialties and Disciplines Link in PCPHC?

- The Role of Psychiatry in PCPHC

- The Role of Gynecology and Obstetrics in PCPHC

- The Role of Public Health in PCPHC

B. Brief Oral Presentations 1 (TBD) 
C. Work Group Meeting 2: Refining the 2015 Geneva Declaration

4:30 - 4:45 PM Coffee Break

4:45 - 6:45 PM General Assembly of the International College of Person-centered Medicine

7:00 - 10:00PM $\quad$ Conference Dinner

CORE CONFERENCE SECOND DAY, TUESDAY APRIL 28, 2015 Geneva University Hospital Marcel Jenny Auditorium and Auxiliary Halls

9:00 AM - 10:30 AM Plenary Symposium 3: Health Services Organization to Achieve Person- and PeopleCentered Primary Health Care

- The Role of the WHO in Achieving Person- and People-Centered Primary Health Care

- The Ethical Framework for Person-Centered Care

- What Do People Want and Expect of PCPHC?

- What is the Nursing Profession Contribution to PCPHC?

- What Should a Nation's Commitment be to PCPHC?

10:30 - 10:45 AM Coffee Break

10:45 AM - 12:15 PM Plenary Symposium 4: Collaborative Interdisciplinary Professional Training for PCPHC

- Inter-Professional Training in PCPHC

- The Integration of Primary Care and Public Health: The Journey of Asclepius and Hygeia

- How Family Medicine Uses a Collaborative Interdisciplinary Training Model

- The Integration of Behavioral Health/Psychiatry and Primary Care

- New Models of Collaborative Training for Resource Challenged Areas

12:15 - 1:15 PM $\quad$ Lunch (open)

1:15 - 2:45 PM Parallel Sessions 3:

A. Panel on Person-Centered Mental Health Contributions to Primary Care (World Psychiatric Association Sections)

B. Shared Decision Making (SDM)

- The Framework of SDM

- European Experience with SDM

- North American Experience with SDM

- African experience with SDM

C. Workgroup Meeting: Person Centered Clinical Care Guiding Principles

2:45 - 3:00 PM Coffee Break

3:00 - 4:30 PM Parallel Sessions 4:

A. Panel on Mental Health and Cultural Perspectives for Primary care

- World Psychiatric Association Perspectives

- World Federation for Mental Health Perspectives

- Francophone Observatory for Person Centered Medicine Perspectives

- World Association for Dynamic Psychiatry (WADP) Perspectives

- International Francophone Psychiatric Federation (ALFAPSY) Perspectives

B. Well-Being Promotion

- Teaching Well Being to Residents and Health Students 
- Patients' Well-being

- Physicians Well Being and Resilience

- Person Centered Prevention

4:30 - 4:45 PM $\quad$ Coffee Break

4:45 - 6:15 PM Parallel Sessions 5:

A. Advocacy and Leadership Policy in Primary Care

- Africa \& Middle East

- Europe

- Americas

- Asia

- Latin America

B. Brief Oral Presentations 2: (TBD)

C. Work Group Meeting: Person Centered Diagnosis

7:00 PM

Dinner (On Your Own with suggested networking opportunities)

\section{CORE CONFERENCE THIRD DAY, WEDNESDAY April 29, 2015}

WHO Headquarters Salle A

8:30 AM - 9:00 AM Key Note Lecture:

Ensuring Health Equity

9:00 AM - 10:30 AM Plenary Symposium 5: Research Priorities for PCPHC

- Research Priorities for Person-centered Conceptualization)

- Research Priorities for Person-centered Measurement

- Research Priorities for Social Determinants of Care

- Research Priorities for Person Centered Primary Care

- Research Priorities on Care Processes and Decision Making

10:30 -10:45 AM Coffee Break

10:45 AM - 11:45 AM Plenary Closing Session:

- The Winds of Change: The Essential Role of Primary Care and Family Medicine in Meeting the Changing Healthcare Needs of Society

- 2015 Geneva Declaration

- Closing Remarks

11:45-12:00M Break

12:00 - 2:00PM WHO Special Session: Collaborating towards the implementation of WHO's Strategy on People-centered and Integrated Services

- Report on the WHO Strategy

- Contributions from Collaborating Organizations

- Next Steps 